\title{
On Microstructure Evolution in Fiber-Reinforced Elastomers and Implications for Their Mechanical Response and Stability
}

Oscar Lopez-Pamies

Department of Mechanical Engineering,

State University of New York,

Stony Brook, NY 11794-2300

e-mail: oscar.lopez-pamies@sunysb.edu

Martín I. Idiart

Área Departamental Aeronáutica,

Facultad de Ingeniería,

Universidad Nacional de La Plata,

Calles 1 y 47

La Plata B1900TAG, Argentina;

Consejo Nacional de Investigaciones Científicas

y Técnicas (CONICET),

Avenida Rivadavia 1917,

Ciudad de Buenos Aires C1033AAJ, Argentina

e-mail: martin.idiart@ing.unlp.edu.ar

Zhiyun Li

Department of Mechanical Engineering,

State University of New York,

Stony Brook, NY 11794-2300
Lopez-Pamies and Idiart (2010, "Fiber-Reinforced Hyperelastic Solids: A Realizable Homogenization Constitutive Theory," J. Eng. Math., 68(1), pp. 57-83) have recently put forward a homogenization theory with the capability to generate exact results not only for the macroscopic response and stability but also for the evolution of the microstructure in fiber-reinforced hyperelastic solids subjected to finite deformations. In this paper, we make use of this new theory to construct exact, closed-form solutions for the change in size, shape, and orientation undergone by the underlying fibers in a model class of fiber-reinforced hyperelastic solids along arbitrary $3 D$ loading conditions. Making use of these results, we then establish connections between the evolution of the microstructure and the overall stress-strain relation and macroscopic stability in fiber-reinforced elastomers. In particular, we show that the rotation of the fibers may lead to the softening of the overall stiffness of fiber-reinforced elastomers under certain loading conditions. Furthermore, we show that this geometric mechanism is intimately related to the development of long-wavelength instabilities. These findings are discussed in light of comparisons with recent results for related material systems. [DOI: 10.1115/1.4002642]

Keywords: finite strain, microstructures, instabilities, homogenization, Hamilton-Jacobi equation

\section{Introduction}

Elastomeric materials reinforced by a single family of aligned cylindrical fibers constitute an important class of technological and natural material systems. A classical example is that of tires and prominent examples of more recent interest include nanostructured thermoplastic elastomers $[1,2]$, as well as biological tissues $[3,4]$. It is by now well recognized that at finite deformations, the mechanical response and stability of these materials depend critically on the underlying evolution of microstructure. However, the strong material and geometric nonlinearities inherent to finite deformations have thus far hampered a rigorous and thorough analysis of this phenomenon.

In this paper, our main objective is to construct exact results for the change in (relative) size, shape, and orientation of the underlying fibers in fiber-reinforced elastomers that are subjected to arbitrary 3D finite deformations. Furthermore, we aim at understanding the effect that such an evolution of microstructure has on the mechanical response and stability of these material systems. To this end, we will make use of the iterative homogenization theory recently put forward by Lopez-Pamies and Idiart [5]. A key feature of this theory is that in addition to its principal capability of providing the exact homogenized (or macroscopic) response for large classes of fiber-reinforced hyperelastic solids, it does also grant access to information on the local fields within each constituent (i.e., the matrix and the fibers). It is precisely this feature that will allow us to establish exact relations for the change in size, shape, and orientation of the fibers along arbitrary loading paths.

While the theory proposed in Ref. [5] applies to fairly general classes of materials, in this work, we will focus on the case of

Contributed by the Materials Division of ASME for publication in the JouRnAL of Engineering Materials and Technology. Manuscript received February 17, 2010; final manuscript received April 16, 2010; published online December 1, 2010. Assoc. Editor: Valeria LaSaponara. fiber-reinforced elastomers made up of a neo-Hookean matrix reinforced by a transversely isotropic distribution of stiffer neoHookean fibers. As it will become apparent further below, this case is general enough to illustrate the fundamental connections between the evolution of microstructure and the macroscopic behavior of fiber-reinforced elastomers while permitting, at the same time, explicit mathematical treatment.

The outline of the paper is as follows. Section 2 lays out the basic equations that characterize the mechanical response, macroscopic stability, and microstructure evolution in fiber-reinforced elastomers. Section 3 summarizes the main results of the iterative homogenization theory of Lopez-Pamies and Idiart [5]. In Sec. 4, we make use of the results of Sec. 3 to solve the equations presented in Sec. 2. In particular, we work out closed-form solutions for the macroscopic response, stability, and the change in size, shape, and orientation of the fibers in fiber-reinforced elastomers made up of a neo-Hookean matrix reinforced by a transversely isotropic distribution of stiffer neo-Hookean fibers. The analytical solutions put forward in Sec. 4 are then examined in detail in Sec. 5 for various loading conditions, fiber-to-matrix heterogeneity contrasts, and initial volume fractions of fibers in order to illustrate the main geometric mechanisms by which the evolution of the microstructure affects the macroscopic mechanical behavior of fiber-reinforced elastomers. Finally, in Sec. 6, some concluding remarks are presented.

\section{Problem Formulation}

Consider a fiber-reinforced solid made up of a continuous matrix phase reinforced by a random distribution of aligned cylindrical fibers that are perfectly bonded to the matrix. The characteristic size of the cross section of the fibers is assumed to be much smaller than the size of the solid and the scale of variation of the applied loads. It is further assumed that the random microstructure is statistically uniform, ergodic, and transversely isotropic. We denote by $\Omega_{0}$ the volume occupied by a representative specimen 


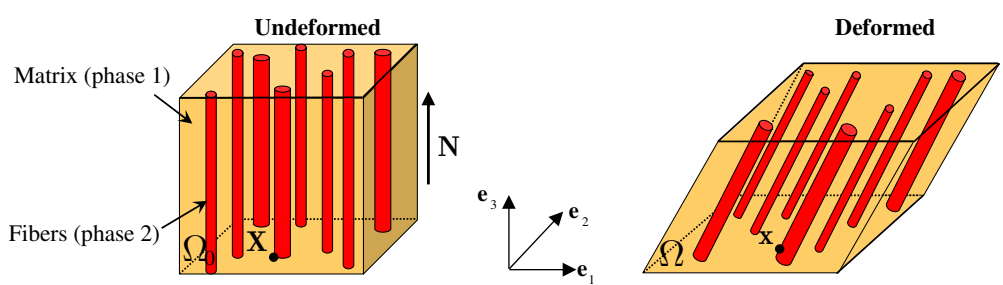

Fig. 1 Schematic illustrating a fiber-reinforced elastomer in the undeformed $\left(\Omega_{0}\right)$ and deformed $(\Omega)$ configurations; note that for convenience, the initial orientation of the fibers $\mathrm{N}$ has been aligned with the coordinate basis vector $e_{3}$

in the undeformed (reference) configuration and by the unit vector $N$ the orientation of the fibers in the undeformed configuration. Upon deformation, the volume occupied by the specimen is denoted by $\Omega$. A material point is identified by its initial position vector $\mathbf{X}$ in $\Omega_{0}$ and by its current position vector $\mathbf{x}$ in $\Omega$ (see Fig. $1)$.

The constitutive response of the matrix $(r=1)$ and fiber $(r=2)$ materials is described in terms of stored-energy functions $W^{(r)}$ that are objective, nonconvex functions of the deformation gradient $\mathbf{F}$. The local constitutive relation can then be conveniently written as

$$
\mathbf{S}=\frac{\partial W}{\partial \mathbf{F}}(\mathbf{X}, \mathbf{F}), \quad W(\mathbf{X}, \mathbf{F})=\left(1-\chi_{0}(\mathbf{X})\right) W^{(1)}(\mathbf{F})+\chi_{0}(\mathbf{X}) W^{(2)}(\mathbf{F})
$$

where $\mathbf{S}$ is the first Piola-Kirchhoff stress tensor and the characteristic function $\chi_{0}$ describes the initial microstructure (i.e., the size, shape, orientation, and location of the fibers in $\Omega_{0}$ ), taking the value 1 if $\mathbf{X}$ is in a fiber and 0 otherwise. Because of the assumed random distribution of the fibers, the dependence of $\chi_{0}$ on $\mathbf{X}$ is not known precisely and the microstructure can only be partially defined in terms of the $n$-point statistics of the system $[6,7]$. Here, use will be made of information up to two-point statistics in order to be able to take advantage of the theory developed in Ref. [5].

Under the above-stated hypotheses, we follow Hill [8] to define the effective stored-energy function ${ }^{1}$ of the fiber-reinforced elastomer as

$$
\bar{W}(\overline{\mathbf{F}})=\min _{\mathbf{F} \in \mathcal{K}^{*}(\overline{\mathbf{F}})} \frac{1}{\Omega_{0}} \int_{\Omega_{0}} W(\mathbf{X}, \mathbf{F}) \mathrm{d} \mathbf{X}
$$

where $\mathcal{K}^{*}$ is a suitably defined set of admissible deformation gradients $\mathbf{F}$ (see Sec. 2 of Ref. [5]). Physically, $\bar{W}$ represents the total elastic energy stored in the entire solid when subjected to an affine displacement boundary condition that is consistent with the average deformation condition $\Omega_{0}^{-1} \int_{\Omega_{0}} \mathbf{F d} \mathbf{X}=\overline{\mathbf{F}}$.

Having formally defined $\bar{W}$, the macroscopic (or homogenized) constitutive relation for the fiber-reinforced elastomer can be written as

$$
\overline{\mathbf{S}}=\frac{\partial \bar{W}}{\partial \overline{\mathbf{F}}}(\overline{\mathbf{F}})
$$

where $\overline{\mathbf{S}}$ denotes the average stress over $\Omega_{0}$ (i.e., $\overline{\mathbf{S}}=\Omega_{0}^{-1} \int_{\Omega_{0}} \mathbf{S d} \mathbf{X}$ ). The effective stored-energy function Eq. (2) also contains information about the macroscopic stability of the material. In particular, it follows from the work of Geymonat et al. [10] that the loss of strong ellipticity of $\bar{W}$ denotes the possible development of

${ }^{1}$ Here, it is relevant to mention that other definitions of macroscopic behavior for hyperelastic composites have been proposed in the literature, including the notion of a "globally equivalent homogeneous" material [9]. long-wavelength instabilities. That is, geometric instabilities with wavelengths that are much larger than the average fiber diameter may develop whenever the condition

$$
B(\overline{\mathbf{F}})=\min _{\|\mathbf{a}\|=\|\mathbf{b}\|=1}\left\{a_{i} b_{j} \overline{\mathcal{L}}_{i j k l}(\overline{\mathbf{F}}) a_{k} b_{l}\right\}>0
$$

ceases to hold true for some applied deformation $\overline{\mathbf{F}}$. In this expression, $\overline{\mathcal{L}}(\overline{\mathbf{F}})=\partial^{2} \bar{W}(\overline{\mathbf{F}}) / \partial \overline{\mathbf{F}}^{2}$ is the effective incremental modulus characterizing the overall incremental response of the material.

The above analysis makes use of a Lagrangian description of the kinematics. The evolution of the microstructure resulting from the finite changes in geometry is thus already accounted for in the homogenized stored-energy function $\bar{W}$. However, even if not necessary to determine the macroscopic constitutive behavior Eq. (3) and stability Eq. (4), it is still of interest to have direct access to variables characterizing the microstructure evolution as they provide deeper insight into the homogenized behavior. For "particulate" material systems like the ones considered here, the microstructural variables of most interest are the volume fraction, average shape, and average orientation of the fibers, all of them in the deformed configuration $[11,12]$. Within the context of the homogenization theory to be utilized in this work, it is possible to write down explicit expressions for such variables (see Sec. 4 of Ref. [5] and Sec. 5 of Ref. [12]). Indeed, the volume fraction of fibers in the deformed configuration is simply given by

$$
c=\frac{\operatorname{det} \overline{\mathbf{F}}^{(2)}}{\operatorname{det} \overline{\mathbf{F}}} c_{0}
$$

where $c_{0}$ is the volume fraction of fibers in the undeformed con-

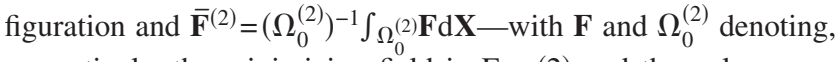
respectively, the minimizing field in Eq. (2) and the volume occupied by the fibers in the undeformed configuration — stands for the average deformation gradient in the fibers. On the other hand, the average shape and average orientation of the fibers are characterized by the principal semiaxes and the principal directions of the Eulerian ellipsoid

$$
E=\left\{\mathbf{x} \mid \mathbf{x} \cdot\left(\mathbf{Z}^{T} \mathbf{Z}\right) \mathbf{x} \leq 1\right\}
$$

where the second-order tensor $\mathbf{Z}$ is defined by

$$
\mathbf{Z}=\mathbf{Z}_{0}\left(\overline{\mathbf{F}}^{(2)}\right)^{-1} \quad \text { with } \mathbf{Z}_{0}=\mathbf{I}-\mathbf{N} \otimes \mathbf{N}
$$

and it is recalled that the unit vector $\mathbf{N}$ indicates the orientation of the cylindrical axis of the fibers in the undeformed configuration. More specifically, the symmetric second-order tensor $\mathbf{Z}^{T} \mathbf{Z}$ has two nonzero eigenvalues $z_{1}$ and $z_{2}$ that serve to define the average elliptical shape of the cross section of the fibers in the deformed configuration. The third eigenvalue $z_{3}$ is zero, indicating that the fibers remain cylindrical (i.e., infinitely long) along any loading path. The eigenvectors $\mathbf{v}_{1}, \mathbf{v}_{2}$, and $\mathbf{v}_{3}$ of $\mathbf{Z}^{T} \mathbf{Z}$ associated with the eigenvalues $z_{1}, z_{2}$, and $z_{3}$ characterize the transverse and cylindrical principal directions of the fibers in the deformed configuration (see Fig. 2). From a computational point of view, it is also worth 


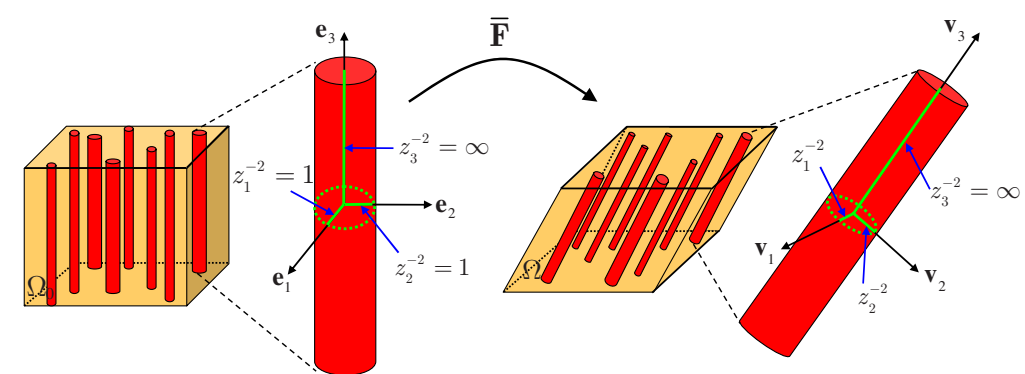

(a)

(b)

Fig. 2 Schematic representation of the evolution of microstructure in a fiber-reinforced elastomer along a loading path with macroscopic deformation gradient $\bar{F}$ : (a) In the undeformed configuration, a typical fiber has a circular cross section (i.e., semiaxes $z_{1}^{-2}=z_{2}^{-2}=1$ ) and its cylindrical axis (with semiaxis $z_{3}^{-2}=\infty$ ) is aligned with the $\mathrm{N}=\mathrm{e}_{3}$ direction. (b) In the deformed configuration, the orientation of the fibers evolves to $v_{3}$, the eigenvector associated with the zero eigenvalue $\left(z_{3}\right)$ of $Z^{T} Z$. In addition, the initial circular cross section evolves into an elliptical cross section with semiaxes and principal directions that are characterized by the eigenvalues $z_{1}$ and $z_{2}$ and corresponding eigenvectors $v_{1}$ and $v_{2}$, of $Z^{\top} Z$ (see Eqs. (6) and (7)).

remarking that the evolution of the volume fraction, average shape, and average orientation of the fibers as characterized by relations (5)-(7) require only knowledge of the average deformation gradient tensor $\overline{\mathbf{F}}^{(2)}$ in the fibers.

In summary, the above-laid-out equations formally characterize the constitutive response, macroscopic stability, and evolution of microstructure of a large class of fiber-reinforced elastomers. It is important to emphasize, however, that the computation of $\bar{W}$ and $\overline{\mathbf{F}}^{(2)}$ is, in general, a hopelessly difficult task because of the nonconvexity of $W^{(1)}$ and $W^{(2)}$ and the randomness of the distribution of fibers as characterized by $\chi_{0}$. Over the past ten years, substantial progress has been made in the development of variational techniques to construct robust approximate solutions (see, e.g., Refs. [12-15] and references therein). More recently, LopezPamies and Idiart [5] have worked out an iterative homogenization method that is actually capable of generating exact solutions for $\bar{W}$ and $\overline{\mathbf{F}}^{(2)}$. In Sec. 3, for convenience and clarity, we summarize the basic elements of this theory that are needed for our purposes.

\section{The Iterative Homogenization Method}

In order to generate exact solutions for $\bar{W}$ and $\overline{\mathbf{F}}^{(2)}$, LopezPamies and Idiart [5] have proposed a "realizable" strategy. Roughly speaking, the idea is to construct a special but yet sufficiently general family of random microstructures that permit the exact computation of the resulting homogenization problem. The strategy comprises two main steps: (i) the first step [16] consists of an iterated homogenization procedure (or differential scheme) that provides an exact solution for $\bar{W}$ in terms of an auxiliary dilute problem; (ii) the second step $[17,18]$ deals with the auxiliary dilute problem, which consists in the construction of suitable classes of sequential laminates. The combination of these two steps (see Sec. 3.3 of Ref. [5]) leads to an explicit framework for generating solutions for the total elastic energy $\bar{W}$ and the local fields including $\overline{\mathbf{F}}^{(2)}$ directly in terms of $W^{(1)}$ and $W^{(2)}$, the oneand two-point statistics of the random distribution of fibers, and the applied loading conditions $\overline{\mathbf{F}}$. To conclude these brief introductory remarks, it is appropriate to point out that because of the iterative construction process, the microstructures that this method generates can be best interpreted as containing fibers with crosssectional areas of infinitely diverse sizes.
For the transversely isotropic fiber-reinforced elastomers of interest in this work, the iterated homogenization result for the effective stored-energy function $\bar{W}=\bar{W}\left(\overline{\mathbf{F}}, c_{0}\right)$ is implicitly determined by the following first-order nonlinear partial differential equation (PDE):

$$
c_{0} \frac{\partial \bar{W}}{\partial c_{0}}-\bar{W}-\max _{\omega \in \mathbb{R}^{3}} \int_{0}^{2 \pi} \frac{1}{2 \pi}\left[\omega_{i} \frac{\partial \bar{W}}{\partial \bar{F}_{i j}} \xi_{j}-W^{(1)}(\overline{\mathbf{F}}+\boldsymbol{\omega} \otimes \boldsymbol{\xi})\right] \mathrm{d} \theta=0
$$

subject to the initial condition

$$
\bar{W}(\overline{\mathbf{F}}, 1)=W^{(2)}(\overline{\mathbf{F}})
$$

Similarly, the average deformation gradient $\overline{\mathbf{F}}^{(2)}=\overline{\mathbf{F}}^{(2)}\left(\overline{\mathbf{F}}, c_{0}\right)$ in the fibers is implicitly determined by the following linear PDE:

$$
c_{0} \frac{\partial \bar{F}_{i j}^{(2)}}{\partial c_{0}}-\frac{\partial \bar{F}_{i j}^{(2)}}{\partial \bar{F}_{k l}} \int_{0}^{2 \pi} \frac{1}{2 \pi} \omega_{k} \xi_{l} \mathrm{~d} \theta=0
$$

where $\omega$ is the maximizing vector in Eq. (8) subject to the initial condition

$$
\bar{F}_{i j}^{(2)}(\overline{\mathbf{F}}, 1)=\bar{F}_{i j}
$$

In the above expressions, $\xi_{1}=\cos \theta, \xi_{2}=\sin \theta$, and $\xi_{3}=0$, Latin indices ranging from 1 to 3 , the usual summation convention is employed, and all of the vector and tensor components are referred without loss of generality to a coordinate system $\mathbf{e}_{i}(i$ $=1,2,3$ ) with $\mathbf{N}=\mathbf{e}_{3}$ (see Fig. 1).

For a detailed description of the derivation and of the various quantities involved in the above relations, we refer to Ref. [5]. In the present context, it is appropriate to mention that the nonlinear first-order PDE (8) corresponds to a Hamilton-Jacobi equation, where the fiber concentration $c_{0}$ and the macroscopic deformation gradient $\overline{\mathbf{F}}$ play the role of "time" and "space" variables, respectively (see, e.g., Chapter 14 of Ref. [19]). Due to the prominent role of the Hamilton-Jacobi equations in physics, a substantial body of literature exists on efficient techniques for solving this type of equations (see, e.g., Refs. $[19,20]$ ). Hence, in spite of its generality, expression (8) is fairly tractable and thus expected to be extremely useful for generating analytical results. A similar comment applies to the linear first-order PDE (10) for $\overline{\mathbf{F}}^{(2)}$, which 
could be solved by a variety of techniques such as, for instance, the standard method of characteristics.

\section{Closed-Form Solutions for Fiber-Reinforced Neo- Hookean Elastomers}

The results presented in Sec. 3 are valid for any behavior of the matrix and fiber phases as characterized by the stored-energy functions $W^{(1)}$ and $W^{(2)}$. The aim of this work is to make use of these results for the first time to investigate the effect of microstructure evolution on macroscopic behavior and stability. In this regard, attention will be restricted to a specific case that is general enough to contain all of the essential features of the problem and that at the same time leads to closed-form solutions. Thus, in this section, we will concentrate on the class of fiber-reinforced elastomers made up of an incompressible neo-Hookean matrix phase reinforced by a transversely isotropic distribution of incompressible stiffer neo-Hookean fibers. The stored-energy functions characterizing both phases are given by

$$
W^{(r)}(\mathbf{F})=\frac{\mu^{(r)}}{2}\left(I_{1}-3\right)
$$

$(r=1,2)$, where the positive material constants $\mu^{(1)}$ and $\mu^{(2)}$ correspond to the shear moduli of the matrix and fibers in the ground state and $I_{1}=\mathbf{F} \cdot \mathbf{F}$ stands for the first principal invariant associated with $\mathbf{F}$.

Within a slightly more general context, the specialization of Eq. (8) for $\bar{W}$ to the case of neo-Hookean phases (Eq. (12)) has already been worked out in Sec. 6 of Ref. [5]. In the sequel (Sec. 4.1), we recall the relevant results from that work and utilize them to write down closed-form expressions for the corresponding overall constitutive response (3) and macroscopic stability condition (4). In Sec. 4.2 further below, we construct a closed-form solution of Eq. (10) for $\overline{\mathbf{F}}^{(2)}$ when specialized to neo-Hookean matrix and fiber phases (Eq. (12)). This solution is, in turn, utilized to construct explicit expressions, with the help of relations (5)-(7), for the change in volume fraction, shape, and orientation of the underlying fibers in fiber-reinforced neo-Hookean elastomers along arbitrary loading paths. We conclude this section by further specializing the solutions derived in Secs. 4.1 and 4.2 to the practically relevant case of nearly rigid fibers (i.e., $\mu^{(2)}$ $\left.\gg \mu^{(1)}\right)$.

4.1 Overall Constitutive Behavior and Stability. Because of the isotropy and incompressibility of the local constitutive behaviors (Eq. (12)) together with the transversely isotropic distribution of fibers, it is first helpful to recognize that the effective stored-energy function $\bar{W}$ for fiber-reinforced neo-Hookean elastomers depends on $\overline{\mathbf{F}}$ and $\mathbf{N}$ only through a set of four invariants. In this work, we will use the following "canonical" set [21]:

$$
\begin{gathered}
\bar{I}_{1}=\overline{\mathbf{F}} \cdot \overline{\mathbf{F}}, \quad \bar{I}_{2}=\frac{1}{2}\left(\overline{\mathbf{F}} \cdot \overline{\mathbf{F}}-\left(\overline{\mathbf{F}}^{T} \overline{\mathbf{F}}\right) \cdot\left(\overline{\mathbf{F}}^{T} \overline{\mathbf{F}}\right)\right) \\
\bar{I}_{4}=\overline{\mathbf{F}} \mathbf{N} \cdot \overline{\mathbf{F}} \mathbf{N}, \quad \bar{I}_{5}=\overline{\mathbf{F}}^{T} \overline{\mathbf{F}} \mathbf{N} \cdot \overline{\mathbf{F}}^{T} \overline{\mathbf{F}} \mathbf{N}
\end{gathered}
$$

where it is emphasized that the third invariant $\bar{J}=\operatorname{det} \overline{\mathbf{F}}=1$ due to the incompressibility of the matrix and fibers.

Next, given the linear dependence of Eq. (12) on $I_{1}$, it is straightforward to compute (see Appendix C of Ref. [5]) the maximizing vector $\omega$ in Eq. (8). In turn, it is possible to carry out the orientational integral in Eq. (8) and to finally solve the PDE for $\bar{W}$ (see Sec. 6 of Ref. [5]). The solution reads as follows:

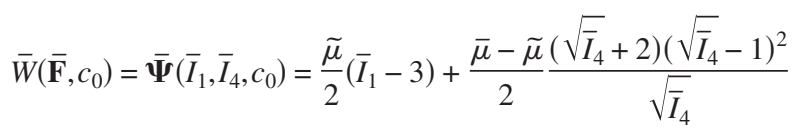

where

$$
\begin{gathered}
\bar{\mu}=\left(1-c_{0}\right) \mu^{(1)}+c_{0} \mu^{(2)}, \\
\tilde{\mu}=\frac{\left(1-c_{0}\right) \mu^{(1)}+\left(1+c_{0}\right) \mu^{(2)}}{\left(1+c_{0}\right) \mu^{(1)}+\left(1-c_{0}\right) \mu^{(2)}} \mu^{(1)}
\end{gathered}
$$

Here, it is worth emphasizing that the effective stored-energy function Eq. (14) is of the separable form $\bar{W}=\bar{W}_{\text {iso }}\left(\bar{I}_{1}\right)+\bar{W}_{\text {fib }}\left(\bar{I}_{4}\right)$ - a functional form, which has been assumed in the literature on a purely phenomenological basis (see, e.g., Refs. [22-24] and references therein) — and that it does not depend on the second $\bar{I}_{2}$ nor fifth $\bar{I}_{5}$ invariants.

Having generated the explicit expression (14) for $\bar{W}$, the overall stress-strain relation for fiber-reinforced neo-Hookean elastomers can then be readily worked out as

$$
\overline{\mathbf{S}}=\frac{\partial \bar{W}}{\partial \overline{\mathbf{F}}}\left(\overline{\mathbf{F}}, c_{0}\right)-\bar{p} \overline{\mathbf{F}}^{-T}=\tilde{\mu} \overline{\mathbf{F}}+(\bar{\mu}-\tilde{\mu})\left(1-\bar{I}_{4}^{3 / 2}\right) \overline{\mathbf{F}} \mathbf{N} \otimes \mathbf{N}-\bar{p} \overline{\mathbf{F}}^{-T}
$$

where the scalar $\bar{p}$ is an arbitrary hydrostatic pressure associated with the macroscopic incompressibility constraint $\operatorname{det} \overline{\mathbf{F}}=1$. Similarly, the corresponding incremental modulus tensor can be easily computed to take the form (in indicial notation)

$$
\begin{aligned}
\overline{\mathcal{L}}_{i j k l}= & \frac{\partial^{2} \bar{W}}{\partial \bar{F}_{i j} \partial \bar{F}_{k l}}\left(\overline{\mathbf{F}}, c_{0}\right)=\tilde{\mu} \delta_{i k} \delta_{j l}+(\bar{\mu}-\tilde{\mu})\left(1-\bar{I}_{4}^{-3 / 2}\right) \delta_{i k} N_{j} N_{l} \\
& +3(\bar{\mu}-\tilde{\mu}) \bar{I}_{4}^{-5 / 2} \bar{F}_{i r} \bar{F}_{k s} N_{r} N_{j} N_{s} N_{l}
\end{aligned}
$$

Upon direct use of Eq. (17) and some algebraic manipulation, the macroscopic stability condition (4) can be shown to simplify to

$$
\begin{aligned}
B(\overline{\mathbf{F}}) & =\min _{\|\mathbf{a}\|=\|\mathbf{b}\|=1}\left\{\tilde{\mu}+(\bar{\mu}-\tilde{\mu})(\mathbf{N} \cdot \mathbf{b})^{2}\left[1-\bar{I}_{4}^{-3 / 2}+3 \bar{I}_{4}^{-5 / 2}\left(\mathbf{N} \cdot \overline{\mathbf{F}}^{T} \mathbf{a}\right)^{2}\right]\right\} \\
& \mathbf{a} \cdot \overline{\mathbf{F}}^{-T} \mathbf{b}=0 \\
& >0
\end{aligned}
$$

where the unit vectors $\mathbf{a}$ and $\mathbf{b}$ are required to satisfy the (incremental) incompressibility constraint $\mathbf{a} \cdot \overline{\mathbf{F}}^{-T} \mathbf{b}=0$. With the help of the generic results put forward in Sec. 5.4 of Ref. [5], it is not difficult to show that along an arbitrary loading path with starting point $\overline{\mathbf{F}}=\mathbf{I}$, the macroscopic stability condition (18) first ceases to hold true at critical deformations $\overline{\mathbf{F}}_{\text {cr }}$ with

$$
\bar{I}_{4}^{\mathrm{cr}}=\overline{\mathbf{F}}_{\mathrm{cr}} \mathbf{N} \cdot \overline{\mathbf{F}}_{\mathrm{cr}} \mathbf{N}=\left(1-\frac{\tilde{\mu}}{\bar{\mu}}\right)^{2 / 3}
$$

As discussed in Sec. 6.1 of Ref. [5] (see also Ref. [25]), the critical condition (19) has a direct physical interpretation. Indeed, the fourth invariant $\bar{I}_{4}$ is a measure of the applied stretch along the fiber direction. Since $\tilde{\mu} \leq \bar{\mu}$, condition (19) plainly states that macroscopic instabilities may develop in fiber-reinforced neoHookean elastomers whenever the compressive stretch along the fibers reaches the critical value $\bar{I}_{4}^{\mathrm{cr}}=(1-\tilde{\mu} / \bar{\mu})^{2 / 3} \leq 1$.

4.2 Microstructure Evolution. Expressions (16) and (19) provide rigorous results that completely characterize the macroscopic constitutive response and stability of fiber-reinforced neoHookean elastomers subjected to finite deformations. In this subsection, with the aim of gaining a more fundamental understanding on the behavior of these materials, we work out results describing the evolution of the volume fraction, shape, and orientation of the underlying fibers along arbitrary loading paths.

We begin by solving Eq. (10) for the average deformation gradient in the fibers $\overline{\mathbf{F}}^{(2)}$. For the case of neo-Hookean constituents (Eq. (12)), the vector $\omega$ in Eq. (10) takes a relatively simple form that allows to compute the integral in Eq. (10) analytically (see the 
Appendix). Once all of the coefficients (i.e., $\left.1 / 2 \pi \int_{0}^{2 \pi} \omega_{k} \xi_{l} \mathrm{~d} \theta\right)$ are known explicitly, the linear PDE (10) can be solved in closedform. The solution reads as follows:

$$
\begin{aligned}
\overline{\mathbf{F}}^{(2)}\left(\overline{\mathbf{F}}, c_{0}\right)= & \gamma_{1}[\overline{\mathbf{F}}-\overline{\mathbf{F}} \mathbf{N} \otimes \mathbf{N}]-\frac{2 \check{\nu}-\gamma_{1}}{{\sqrt{\bar{I}_{4}}}_{4}}\left[\overline{\mathbf{F}}^{-T}-\overline{\mathbf{F}}^{-T} \mathbf{N} \otimes \mathbf{N}\right] \\
& +\frac{2 \check{\nu}-\gamma_{1}}{\bar{I}_{4}} \overline{\mathbf{F}} \mathbf{N} \otimes \mathbf{u}+\overline{\mathbf{F}} \mathbf{N} \otimes \mathbf{N}
\end{aligned}
$$

where

$$
\mathbf{u}=(\mathbf{I}-\mathbf{N} \otimes \mathbf{N}) \overline{\mathbf{F}}^{T} \overline{\mathbf{F}} \mathbf{N}
$$

$\gamma_{1}$ is a scalar function of the principal invariants $\bar{I}_{1}, \bar{I}_{4}$, and $\bar{I}_{5}$ given by

$$
\gamma_{1}=\check{\nu}+\frac{\sqrt{\sqrt{\bar{I}_{4}}+\check{\nu}^{2}\left(\bar{I}_{1} \bar{I}_{4}-\bar{I}_{5}-2 \sqrt{\bar{I}_{4}}\right)}}{\sqrt{\bar{I}_{1} \bar{I}_{4}-\bar{I}_{5}+2 \sqrt{\bar{I}_{4}}}}
$$

and

$$
\check{\nu}=\frac{\mu^{(1)}}{\left(1+c_{0}\right) \mu^{(1)}+\left(1-c_{0}\right) \mu^{(2)}}
$$

Here, it is fitting to remark that Eq. (20) is, of course, a transversely isotropic function of $\overline{\mathbf{F}}$ with symmetric axis $\mathbf{N}$, namely, $\overline{\mathbf{F}}^{(2)}\left(\overline{\mathbf{F}} \mathbf{Q}_{\mathbf{N}}, c_{0}\right)=\overline{\mathbf{F}}^{(2)}\left(\overline{\mathbf{F}}, c_{0}\right) \mathbf{Q}_{\mathbf{N}}$ for all proper orthogonal tensors $\mathbf{Q}_{\mathbf{N}}$ such that $\mathbf{Q}_{\mathbf{N}} \mathbf{N}=\mathbf{N}$ (see, e.g., Ref. [26]). Note also that $\operatorname{det} \overline{\mathbf{F}}^{(2)}\left(\overline{\mathbf{F}}, c_{0}\right)=1$ for all applied deformations $\overline{\mathbf{F}}$ (with $\operatorname{det} \overline{\mathbf{F}}=1$ ) and initial volume fraction of fibers $c_{0}$ as a consequence of the incompressibility of the fibers.

We are now in a position to utilize the solution (20) in the general equations (5)-(7) to establish rigorous relations for the evolution of microstructure in fiber-reinforced neo-Hookean elastomers. Because both the matrix and fibers are incompressible, $\operatorname{det} \overline{\mathbf{F}}=\operatorname{det} \overline{\mathbf{F}}^{(2)}=1$ and therefore Eq. (5) reduces trivially to $c=c_{0}$. That is, the volume fraction of fibers in the deformed configuration remains identical to the volume fraction of fibers in the undeformed configuration for the case of interest here.

On the other hand, the second-order tensor $\mathbf{Z}$ introduced in Eq. (7) can be shown to specialize to

$$
\begin{aligned}
\mathbf{Z}= & (\mathbf{I}-\mathbf{N} \otimes \mathbf{N})\left(\overline{\mathbf{F}}^{(2)}\right)^{-1}=(\mathbf{I}-\mathbf{N} \otimes \mathbf{N})\left[\gamma_{1}\left(\overline{\mathbf{F}}^{-1}-\mathbf{N} \otimes \overline{\mathbf{F}}^{-T} \mathbf{N}\right)\right. \\
& \left.-\left(2 \check{\nu}-\gamma_{1}\right) \sqrt{\bar{I}_{4}}\left(\overline{\mathbf{F}}^{T}-\mathbf{N} \otimes \overline{\mathbf{F}} \mathbf{N}\right)+\frac{2 \check{\nu}-\gamma_{1}}{\sqrt{\bar{I}_{4}}} \mathbf{u} \otimes \overline{\mathbf{F}} \mathbf{N}\right]
\end{aligned}
$$

Given Eq. (24), it is not difficult to deduce that the eigenvalues of the symmetric second-order tensor $\mathbf{Z}^{T} \mathbf{Z}$ defining the Eulerian ellipsoid (Eq. (6)) are given by

$$
\begin{aligned}
& z_{3}=0
\end{aligned}
$$

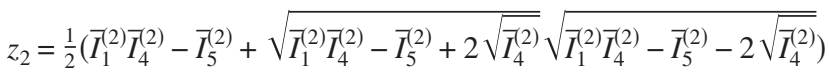

$$
\begin{aligned}
& z_{1}=\frac{1}{2}\left(\bar{I}_{1}^{(2)} \bar{I}_{4}^{(2)}-\bar{I}_{5}^{(2)}-\sqrt{\bar{I}_{1}^{(2)} \bar{I}_{4}^{(2)}-\bar{I}_{5}^{(2)}+2 \sqrt{\overline{I_{4}^{(2)}}}} \sqrt{\left.\bar{I}_{1}^{(2)} \bar{I}_{4}^{(2)}-\bar{I}_{5}^{(2)}-2 \sqrt{\overline{\bar{I}_{4}^{(2)}}}\right)}\right.
\end{aligned}
$$

where

$$
\begin{gathered}
\bar{I}_{1}^{(2)}=\mathbf{F}^{(2)} \cdot \overline{\mathbf{F}}^{(2)}=4 \check{\nu}^{2} \bar{I}_{1}-\left(4 \check{\nu}^{2}-1\right) \frac{2+\bar{I}_{4}^{3 / 2}}{\sqrt{\bar{I}_{4}}} \\
\bar{I}_{4}^{(2)}=\overline{\mathbf{F}}^{(2)} \mathbf{N} \cdot \overline{\mathbf{F}}^{(2)} \mathbf{N}=\bar{I}_{4}
\end{gathered}
$$

$$
\bar{I}_{5}^{(2)}=\left(\overline{\mathbf{F}}^{(2)}\right)^{T} \overline{\mathbf{F}}^{(2)} \mathbf{N} \cdot\left(\overline{\mathbf{F}}^{(2)}\right)^{T} \overline{\mathbf{F}}^{(2)} \mathbf{N}=4 \check{\nu}^{2} \bar{I}_{5}+\left(1-4 \check{\nu}^{2}\right) \bar{I}_{4}^{2}
$$

are principal transversely isotropic invariants of $\overline{\mathbf{F}}^{(2)}$. The eigenvectors $\mathbf{v}_{i}(i=1,2,3)$ of $\mathbf{Z}^{T} \mathbf{Z}$ associated with the above eigenvalues $z_{i}$ can, in turn, be readily computed. They may be written as

$$
\begin{gathered}
\mathbf{v}_{3}=\|\overline{\mathbf{F}} \mathbf{N}\|^{-1} \overline{\mathbf{F}} \mathbf{N} \\
\mathbf{v}_{2}=\left\|\cos \theta_{2} \overline{\mathbf{F}}^{-T} \mathbf{u}+\sin \theta_{2} \overline{\mathbf{F}}^{-T} \mathbf{u}^{\perp}\right\|^{-1}\left(\cos \theta_{2} \overline{\mathbf{F}}^{-T} \mathbf{u}+\sin \theta_{2} \overline{\mathbf{F}}^{-T} \mathbf{u}^{\perp}\right)
\end{gathered}
$$

$$
\mathbf{v}_{1}=\left\|\cos \theta_{3} \overline{\mathbf{F}}^{-T} \mathbf{u}+\sin \theta_{3} \overline{\mathbf{F}}^{-T} \mathbf{u}^{\perp}\right\|^{-1}\left(\cos \theta_{3} \overline{\mathbf{F}}^{-T} \mathbf{u}+\sin \theta_{3} \overline{\mathbf{F}}^{-T} \mathbf{u}^{\perp}\right)
$$

In these last expressions, it is recalled that the vector $\mathbf{u}$ is given by Eq. (21), the vector $\mathbf{u}^{\perp}=\mathbf{N} \times \mathbf{u}$ has been introduced to ease notation and the angles $\theta_{\alpha}$ are given by

$$
\tan \theta_{\alpha}=-\frac{\left[\left(\mathbf{Z}^{T} \mathbf{Z}-z_{\alpha} \mathbf{I}\right) \overline{\mathbf{F}}^{-T} \mathbf{u}\right] \cdot\left[\left(\mathbf{Z}^{T} \mathbf{Z}-z_{\alpha} \mathbf{I}\right) \overline{\mathbf{F}}^{-T} \mathbf{u}^{\perp}\right]}{\left[\left(\mathbf{Z}^{T} \mathbf{Z}-z_{\alpha} \mathbf{I}\right) \overline{\mathbf{F}}^{-T} \mathbf{u}^{\perp}\right] \cdot\left[\left(\mathbf{Z}^{T} \mathbf{Z}-z_{\alpha} \mathbf{I}\right) \overline{\mathbf{F}}^{-T} \mathbf{u}^{\perp}\right]} \quad(\alpha=1,2)
$$

A few remarks regarding the physical significance of the above results are now in order.

- The fact that $\mathbf{Z}^{T} \mathbf{Z}$ has a zero eigenvalue $\left(z_{3}=0\right)$, as already mentioned in Sec. 2, is a general result that applies to any fiber-reinforced elastomer and not just to the fiber-reinforced neo-Hookean elastomers under investigation here. Physically, $z_{3}=0$ implies that the initially cylindrical fibers remain cylindrical (i.e., infinitely long) in the deformed configuration (see Fig. 2). Moreover, the result (27a) for the associated eigenvector $\mathbf{v}_{3}$, which characterizes the average rotation of the cylindrical axes of the fibers along arbitrary loading paths, is also a general result that applies to any fiber-reinforced elastomer. As a direct consequence of its generality, note that $\mathbf{v}_{3}$ depends on the initial microstructure via $\mathbf{N}$ (but not $c_{0}$ ) and the macroscopic deformation gradient

$\overline{\mathbf{F}}$ but not on the constitutive behavior of the phases.

- In connection with the previous remark, it is also interesting to recognize that in the direction of their long axes, the fibers behave as macroscopic material line elements. Indeed, according to the eigenvector $(27 a)$, the direction $\mathbf{N}$ within an undeformed fiber gets mapped to $\overline{\mathbf{F}} \mathbf{N}$ in the deformed configuration. This rigorous result obtained from homogenization therefore supports the popular assumption adopted in phenomenological theories to treat fibers as material line elements (see, e.g., the classical work of Spencer [27]).

- The eigenvalues $z_{1}$ and $z_{2}$, which serve to describe the average (elliptical) shape of the cross section of the fibers in the deformed configuration, are seen to depend rather intricately - as opposed to $z_{3}$ - on the initial microstructure via $\mathbf{N}$ and $c_{0}$, the macroscopic deformation gradient $\overline{\mathbf{F}}$, as well as on the constitutive behavior of the phases $\mu^{(1)}$ and $\mu^{(2)}$. A similar comment applies to the corresponding eigenvectors $\mathbf{v}_{1}$ and $\mathbf{v}_{2}$, which describe the principal directions of the average shape of the cross section of the deformed fibers (see Fig. 2).

4.3 The Case of Nearly Rigid Fibers. The above-presented results correspond to a general heterogeneity contrast between the matrix and the fibers. In practice, however, actual fibers in reinforced soft materials are usually several orders of magnitude stiffer than the matrix phase (i.e., $\left.\mu^{(2)} \gg \mu^{(1)}\right)$. In this regard, let $\Delta \doteq 1 / \mu^{(2)}$ be a small parameter and expand expressions (14), (16), (19), and (20) for the effective stored-energy function, overall stress-strain relation, stability criterion, and average fiber deformation, respectively. The expansions are 


$$
\text { energy: } \begin{aligned}
\bar{W}\left(\overline{\mathbf{F}}, c_{0}\right)= & \frac{c_{0}\left(\sqrt{\bar{I}_{4}}+2\right)\left(\sqrt{\bar{I}_{4}}-1\right)^{2}}{2 \sqrt{\bar{I}_{4}}} \Delta^{-1} \\
& +\frac{1+c_{0}}{2\left(1-c_{0}\right)} \mu^{(1)}\left(\bar{I}_{1}-3\right) \\
& -\frac{\left(3-c_{0}\right) c_{0} \mu^{(1)}\left(\sqrt{\bar{I}_{4}}+2\right)\left(\sqrt{\bar{I}}_{4}-1\right)^{2}}{2\left(1-c_{0}\right)}+O(\Delta)
\end{aligned}
$$

stress-strain: $\quad \overline{\mathbf{S}}=c_{0}\left(1-\bar{I}_{4}^{3 / 2}\right) \overline{\mathbf{F}} \mathbf{N} \otimes \mathbf{N} \Delta^{-1}+\frac{1+c_{0}}{1-c_{0}} \mu^{(1)} \overline{\mathbf{F}}$

$$
-\frac{\left(3-c_{0}\right) c_{0}}{1-c_{0}} \mu^{(1)} \overline{\mathbf{F}} \mathbf{N} \otimes \mathbf{N}-\bar{p} \overline{\mathbf{F}}^{-T}+O(\Delta)
$$

stability criterion: $\quad \bar{I}_{4}^{\mathrm{cr}}=1-\frac{2\left(1+c_{0}\right)}{3\left(1-c_{0}\right) c_{0}} \mu^{(1)} \Delta+O\left(\Delta^{2}\right)$

$$
\text { fiber deformation: } \begin{aligned}
\overline{\mathbf{F}}^{(2)}= & \frac{\bar{I}_{4}^{1 / 4}}{\sqrt{\bar{I}_{1} \bar{I}_{4}-\bar{I}_{5}+2 \sqrt{\bar{I}_{4}}}}\left[\left(\overline{\mathbf{F}}+\frac{1}{\sqrt{\bar{I}_{4}}} \overline{\mathbf{F}}^{-T}\right)\right. \\
& \left.\times(\mathbf{I}-\mathbf{N} \otimes \mathbf{N})-\frac{1}{\bar{I}_{4}} \overline{\mathbf{F}} \mathbf{N} \otimes \mathbf{u}\right]
\end{aligned}
$$$$
+\overline{\mathbf{F}} \mathbf{N} \otimes \mathbf{N}+O(\Delta)
$$

In the limit of rigid fibers as $\Delta \rightarrow 0$, the physical requirement that the energy and stress above remain finite restricts the set of possible deformations to those such that $\bar{I}_{4}=\overline{\mathbf{F}} \mathbf{N} \cdot \overline{\mathbf{F}} \mathbf{N}=1$, namely, deformations that do not involve the stretching of the fibers. For $\Delta=0$, the above expressions then simplify to

$$
\begin{aligned}
\text { energy: } & \bar{W}\left(\overline{\mathbf{F}}, c_{0}\right)=\frac{1+c_{0}}{2\left(1-c_{0}\right)} \mu^{(1)}\left(\bar{I}_{1}-3\right) \\
\text { stress-strain: } \quad & \overline{\mathbf{S}}=\frac{1+c_{0}}{1-c_{0}} \mu^{(1)} \overline{\mathbf{F}}-\bar{q} \overline{\mathbf{F}} \mathbf{N} \otimes \mathbf{N}-\bar{p} \overline{\mathbf{F}}^{-T}
\end{aligned}
$$

stability criterion: $\bar{I}_{4}^{\mathrm{cr}}=1$

$$
\text { fiber deformation: } \quad \overline{\mathbf{F}}^{(2)}=\overline{\mathbf{R}}^{(2)}=\frac{1}{\sqrt{\bar{I}_{1}-\bar{I}_{5}+2}}\left[\left(\overline{\mathbf{F}}+\overline{\mathbf{F}}^{-T}\right)(\mathbf{I}\right.
$$$$
-\mathbf{N} \otimes \mathbf{N})-\overline{\mathbf{F}} \mathbf{N} \otimes \mathbf{u}]+\overline{\mathbf{F}} \mathbf{N} \otimes \mathbf{N}
$$

Several comments are in order. First, the scalar $\bar{q}$ in Eq. (34) is the Lagrange multiplier associated with the macroscopic kinematical constraint $\bar{I}_{4}=\overline{\mathbf{F}} \mathbf{N} \cdot \overline{\mathbf{F}} \mathbf{N}=1$. Second, the critical stretch along the fibers $\bar{I}_{4}^{\mathrm{cr}}$ reduces identically to 1 . This does not imply, however, that macroscopic instabilities in rigidly-reinforced elastomers will develop for all applied deformations $\overline{\mathbf{F}}$ with $\bar{I}_{4}=\overline{\mathbf{F}} \mathbf{N} \cdot \overline{\mathbf{F}} \mathbf{N}=1$, which include, for example, the undeformed configuration. Instead, whether macroscopic instabilities do develop in the limit of rigid fibers depend ultimately on the applied stresses (no instabilities occur, of course, at zero stress), and require, therefore, further analysis which, for conciseness, we will not report here. Third, the average deformation gradient in the fibers reduces to a proper orthogonal second-order tensor $\overline{\mathbf{R}}^{(2)}$ as expected physically since the fibers can only undergo rigid body rotations in this case. Consequently, the tensor $\mathbf{Z}^{T} \mathbf{Z}$ characterizing the average shape and orientation of the fibers in the deformed configuration also simplifies significantly in the rigid-fiber limit. It is easy to show that

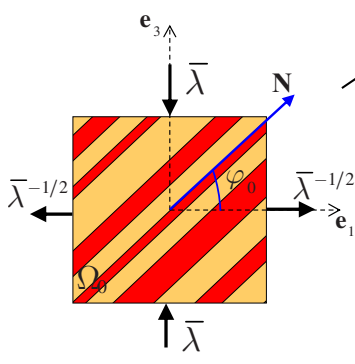

(a)

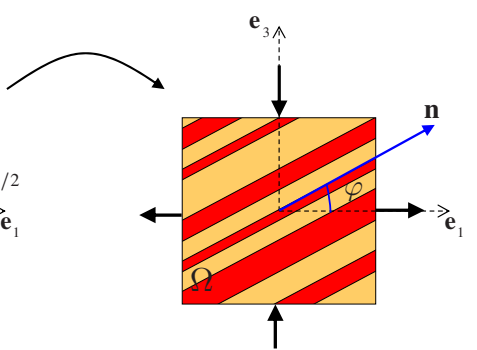

(b)
Fig. 3 Pictorial representation of the applied loading conditions (38) and the resulting evolution of the orientation of the fibers as determined by relations (42) and (43)

$$
\mathbf{Z}^{T} \mathbf{Z}=\overline{\mathbf{R}}^{(2)} \mathbf{Z}_{0}^{T} \mathbf{Z}_{0}\left(\overline{\mathbf{R}}^{(2)}\right)^{T}=\mathbf{I}-\overline{\mathbf{R}}^{(2)}(\mathbf{N} \otimes \mathbf{N})\left(\overline{\mathbf{R}}^{(2)}\right)^{T}
$$

From this result, it is evident that the eigenvalues of $\mathbf{Z}^{T} \mathbf{Z}$ are equal to those of $\mathbf{Z}_{0}^{T} \mathbf{Z}_{0}=(\mathbf{I}-\mathbf{N} \otimes \mathbf{N})$. This implies that the shape of the fibers will remain fixed upon deformation-that is, $z_{1}=z_{2}=1$ and $z_{3}=0-$ which is, of course, consistent with the fibers being rigid. On the other hand, the rotation tensor $\overline{\mathbf{R}}^{(2)}$ serves to fully characterize the reorientation of the principal axes of $\mathbf{Z}^{T} \mathbf{Z}$ with respect to those of $\mathbf{Z}_{0}^{T} \mathbf{Z}_{0}=(\mathbf{I}-\mathbf{N} \otimes \mathbf{N})$.

\section{Sample Results and Discussion}

In order to provide more insight into the analytical solutions of the previous section, we next present illustrative results for specific loading conditions $\overline{\mathbf{F}}$, initial orientation of fibers $\mathbf{N}$, values of fiber-to-matrix heterogeneity contrast $t \doteq \mu^{(2)} / \mu^{(1)}$, and initial volume fraction of fibers $c_{0}$. Of special interest is to bring out the interplay between microstructure evolution and macroscopic constitutive response and stability.

Motivated by possible comparisons with experiments, we consider applied axisymmetric compressive deformations $\overline{\mathbf{F}}$ of the form

$$
\overline{\mathbf{F}}=\bar{\lambda}^{-1 / 2}\left(\mathbf{e}_{1} \otimes \mathbf{e}_{1}+\mathbf{e}_{2} \otimes \mathbf{e}_{2}\right)+\bar{\lambda} \mathbf{e}_{3} \otimes \mathbf{e}_{3}
$$

where $\bar{\lambda}$ is a loading parameter in the range $0<\bar{\lambda} \leq 1$ that takes the value 1 in the undeformed configuration. Moreover, the initial orientation of the cylindrical axes of the fibers is henceforth parameterized as

$$
\mathbf{N}=\cos \varphi_{0} \mathbf{e}_{1}+\sin \varphi_{0} \mathbf{e}_{3}
$$

with the angle $\varphi_{0} \in[0,90 \mathrm{deg}]$ (see Fig. $3(a)$ ). Thus, the case $\varphi_{0}=90 \mathrm{deg}$ corresponds to uniaxial compression along the fibers while $\varphi_{0}=0$ corresponds to a type of transverse uniaxial compression with tensile load being applied along the fibers. Given Eqs. (38) and (39), the scalar overall stress $\bar{S} \doteq-\mathrm{d} \bar{W} / \mathrm{d} \bar{\lambda}$ (which, in this case, proves easier to visualize than the effective stored-energy function $\bar{W}$ and the individual components of $\overline{\mathbf{S}}$ ) is given by

$$
\begin{aligned}
\bar{S}= & -\frac{\mathrm{d} \bar{W}}{\mathrm{~d} \bar{\lambda}}=\tilde{\mu} \frac{1-\bar{\lambda}^{3}}{\bar{\lambda}^{2}}+(\bar{\mu}-\tilde{\mu}) \\
& \times \frac{\left(\cos ^{2} \varphi_{0}-2 \bar{\lambda}^{3} \sin ^{2} \varphi_{0}\right)\left[\left(\cos ^{2} \varphi_{0}+\bar{\lambda}^{3} \sin ^{2} \varphi_{0}\right)^{3 / 2}-\bar{\lambda}^{3 / 2}\right]}{2 \bar{\lambda}^{2}\left(\cos ^{2} \varphi_{0}+\bar{\lambda}^{3} \sin ^{2} \varphi_{0}\right)^{3 / 2}}
\end{aligned}
$$

and the macroscopic stability criterion (19) specializes to 


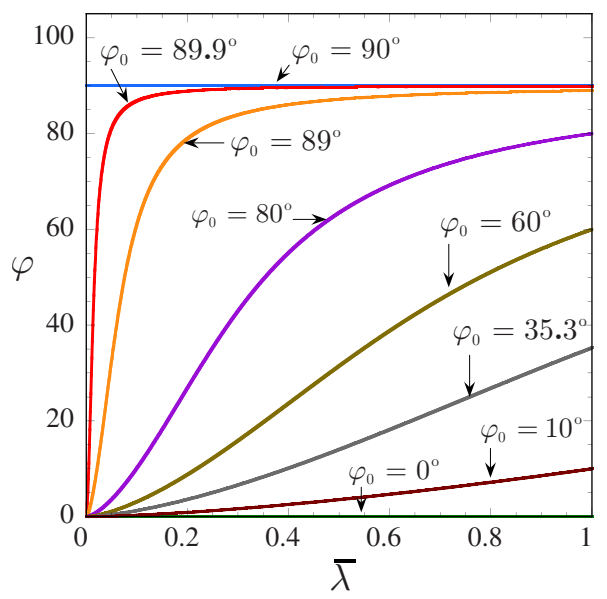

Fig. 4 Angle of rotation of the fibers $\varphi$ (given by expression (43)) in fiber-reinforced neo-Hookean elastomers subjected to axisymmetric compression (Eq. (38)) for various initial fiber orientations $\varphi_{0}$ as a function of the applied macroscopic stretch $\bar{\lambda}$; Note that the results are completely independent of the constitutive behavior of the matrix and fibers, as well as of the volume fraction of fibers

$$
\bar{\lambda}_{\mathrm{cr}}^{3} \sin ^{2} \varphi_{0}-\left(1-\frac{\tilde{\mu}}{\bar{\mu}}\right)^{2 / 3} \bar{\lambda}_{\mathrm{cr}}+\cos ^{2} \varphi_{0}=0
$$

with the critical stretch $\bar{\lambda}_{\text {cr }}$ determining the deformation at which long-wavelength instabilities may first develop along axisymmetric loading paths (Eq. (38)). Furthermore, the average fiber orientation (Eq. (27a)) in the deformed configuration reduces to

$$
\mathbf{n} \doteq \mathbf{v}_{3}=\cos \varphi \mathbf{e}_{1}+\sin \varphi \mathbf{e}_{3}
$$

where

$$
\varphi=\operatorname{Arcos}\left[\frac{\cos \varphi_{0}}{\sqrt{\cos ^{2} \varphi_{0}+\bar{\lambda}^{3} \sin ^{2} \varphi_{0}}}\right]
$$

as depicted in Fig. $3(b)$. The evolution of the remaining microstructural variables $\left(z_{1}, z_{2}, \mathbf{v}_{1}, \mathbf{v}_{2}\right)$ are of lesser importance in the present context of axisymmetric loading conditions and will not be detailed here for conciseness.
We begin by examining the angle of rotation of the fibers $\varphi$ as a function of the applied deformation $\bar{\lambda}$. Results are shown in Fig. 4 for various initial fiber orientations $\left(\varphi_{0}=0,10 \mathrm{deg}, 35.3 \mathrm{deg}, 60\right.$ deg, $80 \mathrm{deg} 89.9^{\circ} \mathrm{deg}$, and $90 \mathrm{deg}$ ). Recall that fiber rotation was found to be independent of matrix and fiber constitutive response and of fiber volume fraction. It is seen that fibers rotate away from the axis of applied compression and toward the axis of applied tension as deformation progresses (i.e., $\varphi \rightarrow 0$ as $\bar{\lambda} \rightarrow 0$ monotonically for all $\left.\varphi_{0} \in[0,90 \mathrm{deg}]\right)$. For the smaller orientations $\varphi_{0}$, fiber rotation evolves gradually with deformation. As $\varphi_{0}$ approaches $90 \mathrm{deg}$, however, fiber rotation is initially negligible but drops rapidly after a certain deformation level. Finally, in the limiting case of $\varphi_{0}=90 \mathrm{deg}$, fibers do not rotate as dictated by the symmetry of the problem. It will be seen below that the ability of the fibers to undergo large and rapid rotations plays a critical role in the macroscopic response and stability of fiber-reinforced elastomers.

The macroscopic response as characterized by relation (40) is shown in Fig. 5. Figure 5(a) displays the overall stiffness $\mathrm{d} \bar{S} /\left.\mathrm{d} \bar{\lambda}\right|_{\lambda=1} ^{-}$in the small-deformation regime as a function of the initial fiber orientation $\varphi_{0}$ for various fiber-to-matrix heterogeneity contrasts $(t=5,20,50)$ and a moderate volume fraction of fibers $\left(c_{0}=30 \%\right)$. In this regime, where no evolution of microstructure takes place, it is seen that the overall stiffness is highest when the fibers are perfectly aligned with the compressive axis of loading $\left(\varphi_{0}=90 \mathrm{deg}\right)$ and is lowest when the fiber orientation is $\varphi_{0}$ $=\operatorname{arcos} \sqrt{2 / 3} \approx 35.3 \mathrm{deg}$ regardless of contrast. These angles do not depend on fiber concentration either. In fact, maximum stiffness along the fibers is a universal feature of fiber-reinforced solids while minimum stiffness at $\varphi_{0} \approx 35.3 \mathrm{deg}$ can be shown to be a feature shared by all transversely isotropic reinforced solids with effective stored-energy functions independent of the invariant $\bar{I}_{5}$, which is the case here.

As deformation progresses $(\bar{\lambda}$ decreases $)$ into the largedeformation regime, the constitutive response turns out to be strikingly different as a result of the underlying microstructure evolution. Figure $5(b)$ displays the overall stress $\bar{S}$ as a function of applied deformation $\bar{\lambda}$ for the choice $t=20, c_{0}=30 \%$, and the various initial fiber orientations considered in Fig. 4. While at small deformation levels, large fiber orientations $\left(\varphi_{0}=60 \mathrm{deg}, 80 \mathrm{deg}\right)$ lead to stiffer responses than small orientations $\left(\varphi_{0}=10 \mathrm{deg}, 35.3\right.$ deg) in accordance with Fig. 5(a); at large deformation levels,
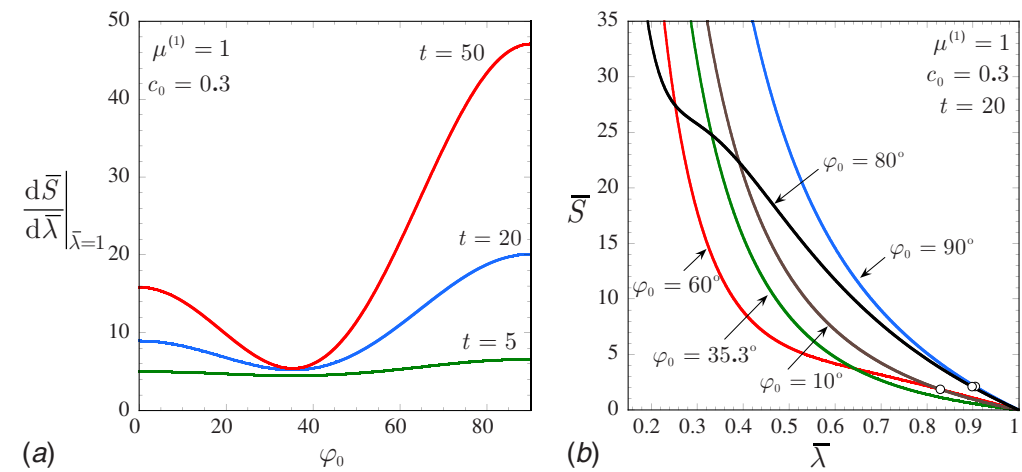

Fig. 5 Overall constitutive response (see Eq. (40)) of fiber-reinforced neo-Hookean elastomers subjected to axisymmetric compression: (a) results for the stiffness $d \bar{S} / d \bar{\lambda}$ in the ground state $(\bar{\lambda}=1)$ for fiber-to-matrix heterogeneity contrasts $t=\mu^{(2)} / \mu^{(1)}=5,20,50$ and volume fraction of fibers $c_{0}=30 \%$ as a function of the initial fiber orientation $\varphi_{0}$ and $(b)$ results for the overall stress $\bar{S}$ for $\varphi_{0}=10 \mathrm{deg}, 35.3 \mathrm{deg}, 60 \mathrm{deg}, 80 \mathrm{deg}, 90$ deg, $t=20$, and $c_{0}=30 \%$ as a function of the applied loading $\lambda^{-}$ 

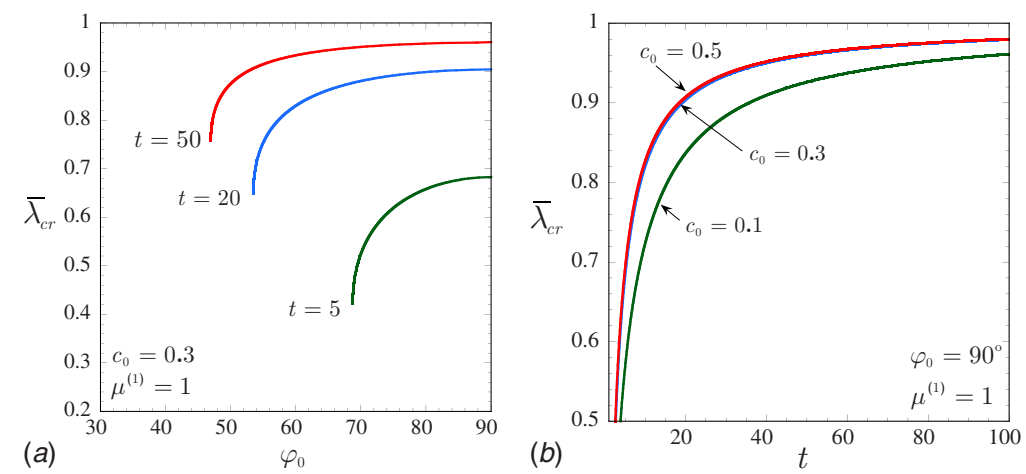

Fig. 6 Onset of macroscopic instabilities (see Eq. (41)) in fiberreinforced neo-Hookean elastomers subjected to axisymmetric compression: (a) results for the critical deformation $\bar{\lambda}_{c r}$ at which instabilities may first develop for fiber-to-matrix heterogeneity contrasts $t=\mu^{(2)} / \mu^{(1)}=5$, 20,50 and volume fraction of fibers $c_{0}=30 \%$ as a function of the initial fiber orientation $\varphi_{0}$ and $(b)$ results for $\bar{\lambda}_{c r}$ for $\varphi_{0}=90$ deg and $c_{0}=10 \%$, $30 \%, 50 \%$ as a function of $t$

they lead to softer responses. The limiting case $\varphi_{0}=90 \mathrm{deg}$, however, remains the stiffest regardless of deformation level. This highly nonlinear dependence of stress on initial fiber orientation is a manifestation of fiber rotation. Small initial orientations induce gradual fiber rotations leading to parabolic responses. Large orientations, by contrast, induce rapid fiber rotations within a small deformation range leading to $\mathrm{S}$-shaped responses with inflexion points precisely where fiber orientations $\varphi$ exhibit a sudden drop, cf. Figs. 4 and 5(b). This strongly suggests that fiber rotation acts as a significant geometric softening mechanism, an interpretation that is consistent with the intuitive notion that a rigid rotation of fibers serves to "accommodate"-at a microscopic length scalepart of the imposed macroscopic deformation at no energy expense.

The symbols " $\circ$ " in Fig. 5(b) denote points at which the fiberreinforced solid becomes unstable as dictated by the criterion (41). Loss of stability is seen to occur for the large fiber orientations only ( $\varphi_{0}=60 \mathrm{deg}, 80 \mathrm{deg}, 90 \mathrm{deg}$ ). This behavior can be linked as well to the rotation of the fibers. The critical stretch $\bar{\lambda}_{\text {cr }}$ at which macroscopic instabilities are first encountered under axisymmetric compressive loadings are displayed in Fig. 6. Figure 6(a) shows $\bar{\lambda}_{\mathrm{cr}}$ for various fiber-to-matrix heterogeneity contrasts $(t=5,20$, 50 ) and fiber volume fraction $c_{0}=30 \%$ as a function of the initial orientation of the fibers $\varphi_{0}$. Figure $6(b)$ shows $\bar{\lambda}_{\mathrm{cr}}$ for $\varphi_{0}$ $=90 \mathrm{deg}$ and fiber volume fractions $c_{0}=10 \%, 30 \%$, and $50 \%$ as a function of the contrast $t$. A key point to remark from Fig. 6(a) is that fiber-reinforced neo-Hookean elastomers are more stable for smaller values of $\varphi_{0}$. In fact, there is a threshold (depending on the contrast $t$ ) in $\varphi_{0}$ beyond which the response remains stable for all applied deformations. Physically, as already mentioned at the end of Sec. 4.1, these results entail that the onset of macroscopic instabilities is governed by the amount of compressive deformation applied along the cylindrical axes of the fibers. Indeed, under loading conditions of the form (38), $\varphi_{0}=90 \mathrm{deg}$ corresponds to the case at which maximum compression is being applied along the fibers. Decreasing the value of $\varphi_{0}$ effectively decreases the amount of compression in the fiber direction. The other major point to recognize from Fig. $6(a)$ is that increasing the contrast between the two phases as measured by the parameter $t$ renders the material more unstable, irrespectively of the volume fraction of the fibers $c_{0}$. This is even more clearly seen in Fig. 6(b).

In connection with all of the results presented in Fig. 6, it is also important to remark that macroscopic stability is consistently lost through the softening of the effective incremental shear response perpendicular to the direction of the cylindrical axes of the fibers. For instance, for the perfectly aligned case of $\varphi_{0}=90 \mathrm{deg}$, the material loses macroscopic stability because of the vanishing of $\overline{\mathcal{L}}_{1313}$ at $\bar{\lambda}_{\text {cr }}$ (see Eq. (4)). This type of "failure" mode is consistent with the development of kink bands, which have been observed to appear in various types of fiber-reinforced materials (see, e.g., Ref. [28]). To better understand these stability results, we turn once more to Fig. 4, where we notice that the more the fibers can potentially rotate-that is, within the present context, the larger the angle $\varphi_{0}$-the more unstable fiber-reinforced neoHookean elastomers become. Physically, this behavior is in accord with the idea that if the fibers - and in particular, the stiff fibersrotate away from the direction of applied compression, the effective incremental shear response of the material in the perpendicular direction to the fibers softens. It is because of this geometric softening that fiber-reinforced neo-Hookean elastomers may become macroscopic unstable in spite of the fact that they are made up of matrix and fibers (Eq. (12)) that are locally stable (i.e., strongly elliptic).

\section{Concluding Remarks}

By exploiting the capability of the theory of Lopez-Pamies and Idiart [5] to generate results for the local (stress and deformation) fields in fiber-reinforced hyperelastic solids, we have been able to derive rigorous closed-form expressions for the evolution in (relative) size, shape, and orientation of the underlying fibers in a representative class of fiber-reinforced elastomers under arbitrary 3D loadings. Exact results of this sort had hitherto been restricted to layered materials (see, e.g., Ref. [29])—the simplest class of composites and a crude 2D idealization of fiber-reinforced solids. The usefulness of these expressions is that they provide a means to identify microscopic mechanisms that govern the macroscopic properties and stability of fiber-reinforced elastomers. In this work, the results have indicated that the rotation of the fiberswhich depends critically on the relative orientation between the loading axes and the fiber direction - can act as a dominant geometric softening mechanism. More specifically, it was found that the long axes of the fibers tend to rotate away from the axis of maximum compressive loading toward the axis of maximum tension. A direct consequence of this behavior is that loadings with predominant compression along the fibers lead to larger rotation of the fibers, which in turn lead to larger geometric softening of the constitutive response and in some cases-when the heterogeneity contrast between the matrix and the fibers is sufficiently high-also to the loss of macroscopic stability. Akin microscopic mechanisms have recently been identified [30,31] in various 
classes of fiber-reinforced elastomers subjected to shear deformations.

We conclude by remarking that the results of this work can help understand the behavior of many other solids with oriented microstructures besides fiber-reinforced elastomers. Indeed, soft modes of deformation and instabilities akin to those reported here have been observed, for instance, in thermoplastic elastomers with lamellar nanostructures under certain types of compressive loadings [32]. Elastomers reinforced with aligned ellipsoidal particles have also been found to exhibit distinctively softer mechanical properties when compressed along the long axes of the particles $[33,34]$. Perhaps even more interestingly, the rapid rotation of aligned mesogens in smectic elastomers has been identified as a key mechanism behind the complex macroscopic properties of this class of liquid crystal elastomers [35].

\section{Appendix: Coefficients in the PDE (10) for the Case of Fiber-Reinforced Neo-Hookean Elastomers}

In this section, we provide explicit expressions for the coefficients $1 / 2 \pi \int_{0}^{2 \pi} \omega_{k} \xi_{l} \mathrm{~d} \theta(k, l=1,2,3)$ that appear in the PDE (10) for $\overline{\mathbf{F}}^{(2)}$ when specialized to the case of fiber-reinforced neoHookean elastomers. Without loss of generality, we write these coefficients in a coordinate system $\mathbf{e}_{i}(i=1,2,3)$, where the initial orientation of the fibers $\mathbf{N}$ is aligned with the coordinate basis vector $\mathbf{e}_{3}$ (see Fig. 1).

$$
\begin{aligned}
\frac{1}{2 \pi} \int_{0}^{2 \pi} \omega_{1} \xi_{2} \mathrm{~d} \theta= & q_{0}\left[\overline{\bar{I}}_{4}\left(q_{2} \bar{F}_{11}+2 q_{1} \bar{F}_{12}\right)+\left(2 q_{1} q_{3}+q_{4}\left(q_{2}-2 \sqrt{\bar{I}_{4}}\right.\right.\right. \\
& \left.\left.\left.-\bar{I}_{1} \bar{I}_{4}+\bar{I}_{5}\right)\right) \bar{F}_{13}\right] \\
\frac{1}{2 \pi} \int_{0}^{2 \pi} \omega_{1} \xi_{2} \mathrm{~d} \theta= & q_{0}\left[\overline{\bar{I}}_{4}\left(2 q_{2} \bar{F}_{11}+q_{2} \bar{F}_{12}\right)+\left(2 q_{1} q_{4}+q_{3}\left(q_{2}-2 \sqrt{\bar{I}_{4}}\right.\right.\right. \\
& \left.\left.\left.-\bar{I}_{1} \bar{I}_{4}+\bar{I}_{5}\right)\right) \bar{F}_{13}\right] \\
& \frac{1}{2 \pi} \int_{0}^{2 \pi} \omega_{1} \xi_{3} \mathrm{~d} \theta=0
\end{aligned}
$$$$
\frac{1}{2 \pi} \int_{0}^{2 \pi} \omega_{3} \xi_{1} \mathrm{~d} \theta=q_{0}\left[\sqrt{\bar{I}_{4}}\left(q_{2} \bar{F}_{21}+2 q_{1} \bar{F}_{22}\right)+\left(2 q_{1} q_{3}+q_{4}\left(q_{2}-2 \sqrt{\bar{I}_{4}}\right.\right.\right.
$$$$
\left.\left.\left.-\bar{I}_{1} \bar{I}_{4}+\bar{I}_{5}\right)\right) \bar{F}_{23}\right]
$$$$
\frac{1}{2 \pi} \int_{0}^{2 \pi} \omega_{3} \xi_{2} \mathrm{~d} \theta=q_{0}\left[\sqrt{\bar{I}_{4}}\left(2 q_{1} \bar{F}_{21}-q_{2} \bar{F}_{22}\right)+\left(2 q_{1} q_{4}+q_{3}\left(-q_{2}\right.\right.\right.
$$$$
\left.\left.\left.-2 \sqrt{\bar{I}_{4}}-\bar{I}_{1} \bar{I}_{4}+\bar{I}_{5}\right)\right) \bar{F}_{23}\right]
$$$$
\frac{1}{2 \pi} \int_{0}^{2 \pi} \omega_{2} \xi_{3} \mathrm{~d} \theta=0
$$$$
\frac{1}{2 \pi} \int_{0}^{2 \pi} \omega_{3} \xi_{1} \mathrm{~d} \theta=q_{0}\left[\sqrt{\bar{I}_{4}}\left(q_{2} \bar{F}_{31}+2 q_{1} \bar{F}_{32}\right)+\left(2 q_{1} q_{3}+q_{4}\left(q_{2}-2 \sqrt{\bar{I}_{4}}\right.\right.\right.
$$$$
\left.\left.\left.-\bar{I}_{1} \bar{I}_{4}+\bar{I}_{5}\right)\right) \bar{F}_{33}\right]
$$$$
\frac{1}{2 \pi} \int_{0}^{2 \pi} \omega_{3} \xi_{2} \mathrm{~d} \theta=q_{0}\left[\sqrt{\bar{I}}_{4}\left(2 q_{1} \bar{F}_{31}-q_{2} \bar{F}_{32}\right)+\left(2 q_{1} q_{4}+q_{3}\left(-q_{2}\right.\right.\right.
$$$$
\left.\left.\left.-2 \sqrt{\bar{I}_{4}}-\bar{I}_{1} \bar{I}_{4}+\bar{I}_{5}\right)\right) \bar{F}_{33}\right]
$$

$$
\frac{1}{2 \pi} \int_{0}^{2 \pi} \omega_{2} \xi_{3} \mathrm{~d} \theta=0
$$

Here,

$$
\begin{gathered}
q_{0}=\frac{\mu^{(1)}-\tilde{\mu}}{2 \mu^{(1)} \sqrt{\bar{I}_{4}}\left(2 \sqrt{\bar{I}_{4}}+\bar{I}_{1} \bar{I}_{4}-\bar{I}_{5}\right)} \\
q_{1}=\bar{C}_{13} \bar{C}_{23}-\bar{C}_{12} \bar{C}_{33} \\
q_{2}=\bar{C}_{13}^{2}-\bar{C}_{23}^{2}-\bar{C}_{11} \bar{C}_{33}+\bar{C}_{22} \bar{C}_{33} \\
q_{3}=\bar{C}_{11} \bar{C}_{23}-\bar{C}_{12} \bar{C}_{13} \\
q_{4}=\bar{C}_{13} \bar{C}_{22}-\bar{C}_{12} \bar{C}_{23}
\end{gathered}
$$

where the notation $\overline{\mathbf{C}}=\overline{\mathbf{F}}^{T} \overline{\mathbf{F}}$ has been used for convenience and it is recalled that the effective constant $\tilde{\mu}$ and principal invariants $\bar{I}_{1}$, $\bar{I}_{4}$, and $\bar{I}_{5}$ are defined, respectively, by Eqs. $(15 b)$ and (13) in the main body of the text.

\section{References}

[1] Honeker, C. C., and Thomas, E. L., 1996, "Impact of Morphological Orientation in Determining Mechanical Properties in Triblock Copolymers," Chem. Mater., 8, pp. 1702-1714.

[2] Honeker, C. C., Thomas, E. L., Albalak, R. J., Hadjuk, D. A., Gruner, S. M., and Capel, M. C., 2000, "Perpendicular Deformation of a Near-Single Crystal Triblock Copolymer With a Cylindrical Morphology. 1. Synchroton SAXS," Macromolecules, 33, pp. 9395-9406.

[3] Finlay, H. M., Whittaker, P., and Canham, P. B., 1998, "Collagen Organization in Branching Region of Human Brain Arteries," Stroke, 29, pp. 1595-1601.

[4] Quapp, K. M., and Weiss, J. A., 1998, "Material Characterization of Human Medial Collateral Ligament," ASME J. Biomech. Eng., 120, pp. 757-763.

[5] Lopez-Pamies, O., and Idiart, M. I., 2010, "Fiber-Reinforced Hyperelastic Solids: A Realizable Homogenization Constitutive Theory,” J. Eng. Math., 68(1), pp. $57-83$.

[6] Willis, J. R., 1981, "Variational and Related Methods for the Overall Properties of Composites," Adv. Appl. Mech., 21, pp. 1-78.

[7] Willis, J. R., 1982, "Elasticity Theory of Composites," Mechanics of Solids, the Rodney Hill 60th Anniversary Volume, H. G. Hopkins, and M. J. Sewell, eds., Pergamon, New York, pp. 653-686.

[8] Hill, R., 1972, "On Constitutive Macrovariables for Heterogeneous Solids at Finite Strain," Proc. R. Soc. London, Ser. A, 326, pp. 131-147.

[9] Chen, Y.-C., Rajagopal, K. R., and Wheeler, L., 2006, "Homogenization and Global Responses of Inhomogeneous Spherical Nonlinear Elastic Shells," J. Elast., 82, pp. 193-214.

[10] Geymonat, G., Müller, S., and Triantafyllidis, N., 1993, "Homogenization of Nonlinearly Elastic Materials, Microscopic Bifurcation and Macroscopic Loss of Rank-One Convexity," Arch. Ration. Mech. Anal., 122, pp. 231-290.

[11] Lopez-Pamies, O., 2006, "On the Effective Behavior, Microstructure Evolution, and Macroscopic Stability of Elastomeric Composites," Ph.D. thesis, University of Pennsylvania.

[12] Lopez-Pamies, O., and Ponte Castañeda, P., 2006, "On the Overall Behavior, Microstructure Evolution, and Macroscopic Stability in Reinforced Rubbers at Large Deformation: I-Theory," J. Mech. Phys. Solids, 54, pp. 807-830.

[13] deBotton, G., Hariton, I., and Socolsky, E. A., 2006, "Neo-Hookean FiberReinforced Composites in Finite Elasticity," J. Mech. Phys. Solids, 54, pp. 533-559.

[14] Agoras, M., Lopez-Pamies, O., and Ponte Castañeda, P., 2009, “A General Hyperelastic Model for Incompressible Fiber-Reinforced Elastomers," J. Mech. Phys. Solids, 57, pp. 268-286.

[15] deBotton, G., and Shmuel, G., "A New Variational Estimate for the Effective Response of Hyperelastic Composites," J. Mech. Phys. Solids, 58, pp. 466483.

[16] Lopez-Pamies, O., 2010, "An Exact Result for the Macroscopic Response of Particle-Reinforced Neo-Hookean Solids," ASME J. Appl. Mech., 77, p. 021016.

[17] Idiart, M. I., 2008, "Modeling the Macroscopic Behavior of Two-Phase Nonlinear Composites by Infinite-Rank Laminates," J. Mech. Phys. Solids, 56, pp. 2599-2617.

[18] deBotton, G., 2005, "Transversely Isotropic Sequentially Laminated Composites in Finite Elasticity," J. Mech. Phys. Solids, 53, pp. 1334-1361.

[19] Polyanin, A. D., Zaitsev, V. F., and Moussiaux, A., 2002, Handbook of First Order Partial Differential Equations, Taylor \& Francis, London.

[20] Benton, S. H., 1977, The Hamilton-Jacobi Equation: A Global Approach. Mathematics in Science and Engineering 131, Academic Press, New York.

[21] Spencer, A. J. M., 1984, Continuum Theory of the Mechanics of FibreReinforced Composites, Springer, New York. 
[22] Merodio, J., and Ogden, R. W., 2003, "Instabilities and Loss of Ellipticity in Fiber-Reinforced Nonlinearly Elastic Solids Under Plane Deformation,” Int. J. Solids Struct., 40, pp. 4707-4727.

[23] Merodio, J., and Ogden, R. W., 2005, "Mechanical Response of FiberReinforced Incompressible Non-Linearly Elastic Solids," Int. J. Non-Linear Mech., 40, pp. 213-227.

[24] Horgan, C. O., and Saccomandi, G., 2005, "A New Constitutive Theory for Fiber-Reinforced Incompressible Nonlinearly Elastic Solids,” J. Mech. Phys. Solids, 53, pp. 1985-2015.

[25] Agoras, M., Lopez-Pamies, O., and Ponte Castañeda, P., 2009, "Onset of Macroscopic Instabilities in Fiber-Reinforced Elastomers at Finite Strain," J. Mech. Phys. Solids, 57, pp. 1828-1850.

[26] Del Piero, G., 1998, "Representation Theorems for Hemitropic and Tranversely Isotropic Tensor Functions," J. Elast., 51, pp. 43-71.

[27] Spencer, A. J. M., 1972, Deformations of Fibre-Reinforced Materials, Oxford University Press, Oxford.

[28] Fleck, N., 1997, "Compressive Failure of Fiber Composites," Adv. Appl. Mech., 33, pp. 43-117.

[29] Lopez-Pamies, O., and Ponte Castañeda, P., 2009, "Microstructure Evolution in Hyperelastic Laminates and Implications for Overall Behavior and Macroscopic Stability," Mech. Mater., 41, pp. 364-374.
[30] Baek, S., and Pence, T. P., 2009, "Emergence and Disappearance of Load Induced Fiber Kinking Surfaces in Transversely Isotropic Hyperelastic Materials," Z. Angew. Math. Phys., 61(4), pp. 745-772.

[31] Destrade, M., Saccomandi, G., and Sgura, I., 2009, "Inhomogeneous Shear of Orthotropic Incompressible Non-Linearly Elastic Solids: Singular Solutions and Biomechanical Interpretation," Int. J. Eng. Sci., 47, pp. 1170-1181.

[32] Lopez-Pamies, O., Garcia, R., Chabert, E., Cavaillé, J.-Y., and Ponte Castañeda, P., 2008, "Multiscale Modeling of Oriented Thermoplastic Elastomers With Lamellar Morphology," J. Mech. Phys. Solids, 56, pp. 32063223.

[33] Wang, S., and Mark, J. E., 1990, "Generation of Glassy Ellipsoidal Particles Within an Elastomer by in Situ Polymerization, Elongation at an Elevated Temperature, and Finally Cooling Under Strain," Macromolecules, 23, pp. 4288-4291.

[34] Lopez-Pamies, O., and Ponte Castañeda, P., 2006, "On the Overall Behavior, Microstructure Evolution, and Macroscopic Stability in Reinforced Rubbers at Large Deformations: II-Application to Cylindrical Fibers," J. Mech. Phys. Solids, 54, pp. 831-863.

[35] Nishikawa, E., and Finkelmann, H., 1999, "Smectic-A Liquid Single Crystal Elastomers-Strain Induced Break-Down of Smectic Layers," Macromol. Chem. Phys., 200, pp. 312-322. 